\section{PREVALENCIA DE SÍNDROME METABÓLICO Y FACTORES DE RIESGO EN LA ETNIA KARIÑA, ESTADO BOLÍVAR, VENEZUELA}

\author{
PREVALENCE OF METABOLIC SYNDROME AND RISK FACTORS KARIÑA \\ ETNIC, BOLIVAR STATE, VENEZUELA
}

Deymar Quiroz' , Deynalia Quiroz' , Francisco J. Bognanno², Melania Marin²

\section{RESUMEN}

Introducción: El Síndrome Metabólico involucra factores de riesgos en conjunto: hipertensión arterial, obesidad, dislipidemia y resistencia a la insulina, que favorecen el desarrollo de enfermedades cerebrovasculares, cardiovasculares y diabetes.

Objetivo general: Determinar la prevalencia del Síndrome Metabólico y sus factores de riesgo en individuos de la etnia Kariña (Mayo 20I3 - Mayo 20I4).

Metodología: Se realizó un estudio observacional, descriptivo y corte transversal; en un universo de 203 individuos; y una muestra de 120 individuos (I8-85 años) de la etnia Kariña de la Comunidad de Mayagua, Estado Bolívar, Venezuela. En todos ellos, el perfil lipídico se analizó mediante el método colorimétrico. La glicemia se cuantificó con glucómetro previo ayuno de 12 horas. Se utilizaron los criterios diagnósticos de la Federación Internacional de Diabetes, Asociación Latinoamericana de Diabetes y Panel III de Tratamiento de Adultos.

Resultados: La prevalencia del síndrome metabólico fue según la Federación Internacional de Diabetes 46,67\%, la Asociación Latinoamericana de Diabetes 39,17\%, y Panel III de Tratamiento de Adultos 38,33\%, el índice de concordancia de Kappa (k) entre Panel III de tratamiento de Adultos y Federación Internacional de Diabetes indica una fuerza de concordancia considerable, dicho índice entre Panel III de Tratamiento de Adultos y Asociación Latinoamericana de Diabetes denota una fuerza de concordancia casi perfecta al igual que el índice entre Asociación Latinoamericana de Diabetes y Federación Internacional de Diabetes.

Conclusión: Se halló alta prevalencia de síndrome metabólico según criterios de Asociación Latinoamericana de Diabetes, Federación Internacional de Diabetes y Panel III de Tratamiento de Adultos; con predominio en el género femenino y en individuos mayores de 50 años.

\section{ABSTRACT}

Metabolic Syndrome includes the association of risk factors: hypertension, obesity, dyslipidemia and insulin resistance, which increases the possibility of developing cerebrovascular, cardiovascular and diabetes diseases.

General objective:To determine the prevalence of the Metabolic Syndrome and its risk factors in individuals of the Kariña ethnic group. May 2013 - May 2014.

Methods: An observational, descriptive study and cross section study was conducted; with a universe of 203 individuals, and a sample of 120 individuals (I8-85 years) of the Kariña ethnic group of the Community of Mayagua, Bolívar State,Venezuela.

In all participants, the lipid profile was analyzed with the colorimetric method. The glycemia was quantified with a glucometer prior to fasting for 12 hours. The diagnostic criteria of the International Diabetes Federation, the Latin American Diabetes Association and the Adult Treatment Panel III were used.

Results: the prevalence of the metabolic syndrome was $46,67 \%$ according to the International Diabetes Federation, 39, I7\% Latin American Diabetes Association, and 38.33\% Adult Treatment Panel II, the Kappa (k) concordance between Adult Treatment Panel III and International Diabetes Federation indicates a considerable concordance force, said Index between Adult Treatment Panel III and Latin American Diabetes Association denotes an almost perfect match strength as does the index between Latin American Diabetes Association and International Diabetes Federation.

Conclusion: A high prevalence of metabolic syndrome was found by both the Latin American Diabetes Association, International Diabetes Federation and Adult Treatment Panel III criteria, with predominance in the female gender and individuals over 50 years old.
1 Médico Cirujano, Escuela de Ciencias de la Salud Dr. "Francisco Battistini Casalta", Universidad de Oriente, Núcleo Bolívar,

2 Médico Internista, Dpto. de Medicina Interna, Hospital Universitario Ruiz y Páez, Ciudad Bolívar, Estado Bolívar, Venezuela.

Correspondencia a: Deymar Quiroz deymardla053@gmail.com Telf. y celular: 0593991608447/ 0593993722775

Palabras clave: obesidad, hiperglicemia y dislipidemia.

Keywords: obesity, hyperglycemia and dyslipidemia.

Procedencia y arbitraje: no comisionado, sometido a arbitraje externo.

Recibido para publicación: 7 de diciembre 2016 Aceptado para publicación: 14 de julio 2018

Citar como: Rev Cient Cienc Med 2018;20(1):7-20

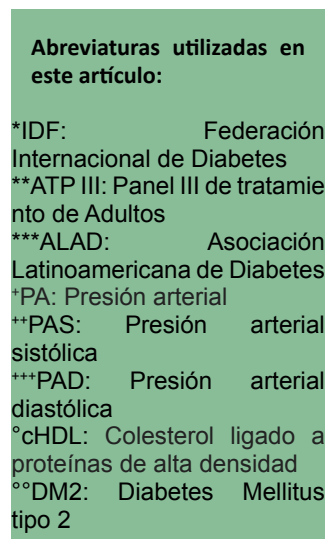




\section{INTRODUCCIÓN}

FI Síndrome Metabólico engloba una asociación de factores de riesgos: hipertensión arterial, obesidad, dislipidemia y resistencia a la insulina (se relaciona con aumento de grasa corporal y ausencia del ejercicio físico), los cuales aumentan la probabilidad de desarrollar enfermedades cerebrovasculares, cardiovasculares y diabetes ${ }^{1-3}$.

En 1998 la Organización mundial de la salud (OMS) describe dicha entidad por la presencia de Diabetes tipo 2, alteración de la tolerancia a la glucosa, resistencia a la insulina o hiperglicemia en ayunas coincidiendo con al menos dos de los siguientes criterios: presión arterial $\geq 140 / 90 \mathrm{mmHg}$; triglicéridos $\geq 150 \mathrm{mg} / \mathrm{dl}$ o colesterol-lipoproteína de alta densidad (HDL) $<35 \mathrm{mg} /$ dl en hombres, $<39 \mathrm{mg} /$ dl en mujeres; índice cintura/cadera $>0,90$ en hombres, $>0,85$ en mujeres o IMC $>30 \mathrm{~kg} / \mathrm{m} 2$; microalbuminuria $\geq 20 \mu \mathrm{g} / \mathrm{min}$ o índice albúmina/creatinina $\geq 30 \mathrm{mg} / \mathrm{g}^{4,5}$. La Asociación Latinoamericana de Diabetes (ALAD) determinó que el perímetro abdominal en varones $>94 \mathrm{~cm}$ y en mujeres $>88 \mathrm{~cm}$ es considerado en el diagnóstico del Síndrome Metabólico, además ha descrito que factores como el estilo de vida, etnia y urbanización influyen en la alta incidencia del síndrome ${ }^{6,7}$. Este síndrome en América Latina se espera que aumente $14 \%$ los siguientes 10 años $^{8}$. En el estado Zulia-Venezuela, un estudio determinó que un cuarto de la población $>20$ años de edad presenta obesidad y un tercio Síndrome Metabólico9. La salud, cultura y sistema tradicional de producción se han modificado por la explotación petrolera y cercanía de dichas poblaciones a ciudades ${ }^{10}$. Debido a que la salud en los grupos indígenas ha cambiado a medida que pasa el tiempo, lo cual se ha asociado a la modificación del estilo de vida por la cercanía a grandes ciudades, además existen pocas investigaciones que documenten sobre dicha entidad clínica en individuos de etnias indígenas venezolanas; se motiva a la elaboración de esta investigación, con la finalidad de determinar la prevalencia de síndrome metabólico en este grupo de individuos.

\section{MATERIALES Y MÉTODOS}

Se llevó a cabo un estudio de tipo observacional, descriptivo y de corte transversal, en la comunidad Mayagua, parroquia Orinoco, municipio Heres, Estado Bolívar-Venezuela. El universo está formado por 203 Kariñas con ascendencia directa entre 18-85 años, de los cuales se tomó una muestra de 120 (59,1\% de la población Kariña en estudio) individuos utilizando la siguiente fórmula:

$\mathrm{n}=\mathrm{N} \sigma^{2} \mathrm{Z}^{2}$

$(\mathrm{N}-1) \mathrm{e}^{2}+\sigma^{2} \mathrm{Z}^{2}$;

donde: $\mathrm{n}=$ tamaño de la muestra; $\mathrm{N}=$ tamaño de la población, $\sigma=$ desviación estándar de la población, se utilizó un valor constante de 0,$5 ; Z=$ niveles de confianza, se empleó el $95 \%$ de confianza que equivale a 1,96; e= límite aceptable de error muestral con un valor de $6 \%(0,06)$.
En los criterios de inclusión se encuentran individuos de la etnia Kariña, género masculino y femenino de ascendencia directa con edades entre 18-85 años. Los criterios de exclusión abarcan indígenas con ascendencia de otro grupo étnico, embarazadas, diagnóstico de enfermedades endocrino-metabólicas: Síndrome Metabólico, Diabetes Mellitus, Síndrome de Cushing.

Los materiales empleados fueron: esfigmomanómetro aneroide (Lane Aneroide CE0123), estetoscopio (Littmann Brand Classic II S.E.), cinta métrica $(1,50 \mathrm{~cm})$, guantes estériles, alcohol isopropílico 70\%, algodón, jeringas de $5 \mathrm{cc}$, scalp n'21, tubos tapa roja, sistema de control de glucosa en sangre SUMASENSOR SXT CE0123, biosensor para glucosa en sangre (SUMASENSOR SXT) y lancetas estériles para uso úniCo (LIANFA 28G).

Para la recolección de datos se utilizó una hoja de consentimiento informado, en la cual se registran datos de identificación, género, etnia, circunferencia de cintura, Presión Arterial Sistólica (PAS) y Presión Arterial Diastólica (PAD).

El perfil lipídico que incluye colesterol total, lipoproteína de Alta Densidad (HDL), Lipoproteína de Baja Densidad (LDL), Lipoproteína de Muy Baja Densidad (VLDL) y triglicéridos se obtuvieron mediante la toma de muestra sanguínea que se analizó con el método colorimétrico. La glicemia se cuantificó con un glucómetro previo ayuno de 12 horas. Se utilizaron los criterios de acuerdo al ATP III, IDF y ALAD (Tabla 1) para determinar la prevalencia de síndrome metabólico ${ }^{11}$.

El análisis estadístico fue realizado utilizando el programa SPSS ${ }^{\circledR} 23$ (versión 23; Statistical Packageforthe Social Sciences, SPSS Inc, Chicago, IL). Se realizaron tablas y figuras por medio del programa Microsoft Excel ${ }^{\circledast}$ 2010. Los datos se analizaron por medio de estadística descriptiva como la media, desviación estándar, valor mínimo, valor máximo, frecuencias absolutas y relativas. La normalidad de las variables fue evaluada aplicando la prueba de Kolmogorov-Smirnov. Debido a que las variables evaluadas no siguieron una distribución normal fueron evaluadas con la prueba Chi cuadrado. El intervalo de confianza fue de $95 \%$ y las diferencias fueron consideradas estadísticamente significativas con $p \leq 0,05$. Para evaluar la concordancia entre los criterios de la ALAD, Federación Internacional de Diabetes (IDF) y Panel III de Tratamiento de Adultos (ATP III) se aplicó el índice de kappa de kohen, y su interpretación se realizó utilizando una escala que expresa cualitativamente la fuerza de concordancia, de acuerdo a Landis y Koch; considerándose: pobre, un coeficiente Kappa (k) de 0,00; leve, un coeficiente Kappa (k) entre 0,01-0,2; aceptable, un coeficiente Kappa (k) entre 0,21-0,40; moderada, un coeficiente Kappa entre (k) 0,410,60; considerable, un coeficiente Kappa (k) entre 0,61-0,80; casi perfecta, un coeficiente Kappa (k) entre 0,81-1,012.

\section{RESULTADOS}

Se obtuvo el valor promedio de edad 42,07 $\pm 16,81$ años, perímetro de cintura $93,88 \pm 14,96 \mathrm{~cm}, \mathrm{HDL} 43,05 \pm 9,66 \mathrm{mg} /$ dl, triglicéridos $130,98 \pm 116,55 \mathrm{mg} / \mathrm{dl}$, glicemia en ayu- 
nas $102,45 \pm 19,03 \mathrm{mg} / \mathrm{dl}$, PAS $120,75 \pm 14,68 \mathrm{mmHg}$ y PAD $81,17 \pm 13,36 \mathrm{mmHg}$.

La frecuencia de obesidad abdominal según la IDF es $77 \%(n=92)$ (género masculino $39 \%[n=47]$ y femenino $38 \%[n=45]$ ), según ALAD $58 \%(n=70)$ (género femenino $31 \%[n=37]$ y masculino $28 \%[n=33])$, según ATPIII $47 \%(n=56)$ (género femenino $31 \%[n=37$ y y masculino $16 \%[n=19]$ ); de acuerdo al género no se presentó diferencia significativa según la IDF y ALAD, mientras que según el ATP III hubo diferencias significativas.

La obesidad abdominal predominó en el grupo de 30-39 años según la IDF con $21,7 \%(n=26)$ y ATPIII $11,7 \%(n=14)$;

Tabla 1. Criterios para el diagnóstico clínico del síndrome metabólico"1. sin presentar diferencias significativas con un valor de $p=0,106$ y $p=0,068$ respectivamente, en cuanto a la ALAD también dominó en dicho grupo etario con $15,8 \%(n=19)$ presentando diferencias significativas $(p=0,025)$.

El HDL bajo en el género femenino, fue $35,8 \%(n=43)$ y en el masculino $25,8 \%(n=31)$ con diferencia significativa. La hipertrigliceridemia fue mayor en el género masculino con 15,8\%, $(n=19)$ (género femenino fue 9,2\%[n=11]); la glicemia en ayuno $\geq 100 \mathrm{mg} / \mathrm{dL}$ en el género femenino $24,2 \%(n=29)$ y en el masculino $23,3 \%(n=28)$, la presión arterial (PAD $\geq 85 \mathrm{mmHg}$ y/o PAS $\geq 130 \mathrm{mmHg}$ ) en el género

\begin{tabular}{|c|c|c|c|c|c|c|}
\hline Parámetros & $\begin{array}{l}\text { Obesidad } \\
\text { Abdominal }\end{array}$ & Triglicéridos altos & $\begin{array}{l}\mathrm{cHDL}^{\circ} \\
\text { Bajos }\end{array}$ & $\mathrm{PA}^{+}$elevada & $\begin{array}{l}\text { Alteración de la } \\
\text { regulación de la } \\
\text { glucosa }\end{array}$ & Diagnóstico \\
\hline IDF $^{*}$ & $\begin{array}{c}\text { Perímetro de } \\
\text { cintura } \geq 90 \mathrm{~cm} \text { en } \\
\text { hombres y } \geq 80 \mathrm{~cm} \\
\text { en mujeres (para } \\
\text { Asia y America } \\
\text { Latina) }\end{array}$ & $\begin{array}{l}>150 \mathrm{mg} / \mathrm{dl} \text { (ó } \\
\text { en tratamiento } \\
\text { hipolipemiante } \\
\text { específico) }\end{array}$ & \multirow{3}{*}{$\begin{array}{c}<40 \mathrm{mg} / \\
\text { dl en } \\
\text { hombres } \\
\text { o }<50 \\
\text { mg/dl en } \\
\text { mujeres ( } \\
\text { tratamiento } \\
\text { con efecto } \\
\text { sobre } \\
\mathrm{cHDL}^{\circ} \text { ) }\end{array}$} & $\begin{array}{c}\mathrm{PAS}^{++} \geq 130 \mathrm{~mm} \mathrm{Hg} \\
\text { y/o } \\
\mathrm{PAD}^{+++} \geq 85 \mathrm{~mm} \mathrm{Hg} \\
\text { o en tratamiento } \\
\text { antihipertensivo }\end{array}$ & $\begin{array}{c}\text { Glucemia en } \\
\text { ayunas } \geq 100 \\
\text { mg/dL o DM2 } \\
\text { diagnosticada } \\
\text { previamente }\end{array}$ & $\begin{array}{l}\text { Obesidad } \\
\text { Abdominal } \\
+2 \text { de los } 4 \\
\text { restantes }\end{array}$ \\
\hline ATP $\| I^{* *}$ & $\begin{array}{c}\text { Perímetro de } \\
\text { cintura }>102 \mathrm{~cm} \\
\text { en hombres (para } \\
\text { hispanos }>94 \mathrm{~cm} \text { ) y } \\
>88 \mathrm{~cm} \text { en mujeres }\end{array}$ & $\begin{array}{l}\geq 150 \mathrm{mg} / \mathrm{dl} \text { (ó } \\
\text { en tratamiento } \\
\text { hipolipemiante } \\
\text { específico) }\end{array}$ & & $>130 / 85 \mathrm{~mm} / \mathrm{Hg}$ & $\begin{array}{c}\text { Glucemia ayunas } \geq \\
100 \mathrm{mg} / \mathrm{dL} \text { o en } \\
\text { tratamiento para } \\
\text { glucemia elevada }\end{array}$ & 3 de los 5 \\
\hline $\mathrm{ALAD}^{* * *}$ & $\begin{array}{c}\text { Perímetro de } \\
\text { cintura } \geq 94 \mathrm{~cm} \text { en } \\
\text { hombres y } \\
\geq 88 \mathrm{~cm} \text { en mujeres }\end{array}$ & $\begin{array}{l}>150 \mathrm{mg} / \mathrm{dl} \text { (ó } \\
\text { en tratamiento } \\
\text { hipolipemiante } \\
\text { específico) }\end{array}$ & & $\begin{array}{l}\mathrm{PAS}^{++} \geq 130 \mathrm{~mm} \mathrm{Hg} \\
\text { y/o } \mathrm{PAD}^{+++} \geq 85 \mathrm{~mm} \\
\mathrm{Hg} \text { o en tratamiento } \\
\text { antihipertensivo }\end{array}$ & $\begin{array}{c}\text { Glucemia Anormal } \\
\text { Ayunas, } \\
\text { Intolerancia a la } \\
\text { glucosa o Diabetes }\end{array}$ & $\begin{array}{c}\text { Obesidad } \\
\text { Abdominal }+2 \text { de } \\
\text { los } 4 \text { restantes }\end{array}$ \\
\hline
\end{tabular}

Fuente: Datos obtenidos del Consenso Latinoamericano de la Asociación Latinoamericana de Diabetes (ALAD) 2010.

masculino $23 \%(n=28)$ y femenino $16 \%(n=19)$, sin presentar diferencias significativas entre género para dichos criterios. (Figura 1)

La prevalencia del síndrome metabólico fue mayor según la IDF $46,67 \%(n=56)$, seguido de la ALAD 39,17\%(n=47), y por último ATP III 38,33\%(n=46). El índice de concordancia de Kappa (k) entre ATP III e IDF fue de 0,797 indicando una fuerza de concordancia considerable; el índice de concordancia de Kappa $(k)$ entre ATP III y ALAD fue de 0,912 denotando una fuerza de concordancia casi perfecta; $y$ el índice de concordancia de Kappa (k) entre ALAD e IDF fue de 0,848 denotando una fuerza de concordancia casi perfecta. (Figura 2)

En cuanto a la prevalencia de síndrome metabólico el género femenino es el más afectado según ALAD 20,83\% $(n=25)$ y ATPIII $20,83 \%(n=25)$, mientras que según la IDF predomina en ambos géneros con $23,33 \%(n=28)$, no se encontraron diferencias significativamente estadísticas.
El Síndrome Metabólico de acuerdo a los grupos etareos presenta diferencias significativas, el más afectado es de $50-59$ años $(9,2 \%[n=11]), 60$ y más años $(9,2 \%[n=11])$ según ALAD $(p=0,045)$ y el ATP III $(p=0,016)$; de acuerdo a la IDF $(p=0,02)$ predomina en el grupo de 30-39 años $(10,8 \%[n=13]), 60$ y más años $(10,8 \%[n=13])$ para cada grupo. (Tabla 2)

\section{DISCUSIÓN}

La edad, composición étnica de las poblaciones, estilo de vida, medio ambiente y estado nutricional entre países pueden tener efectos en la prevalencia de síndrome metabólico ${ }^{13,14}$. En lo que corresponde al perímetro de cintura el valor promedio fue $93,88 \pm 14,96 \mathrm{~cm}$ siendo mayor al de los indígenas de la etnia Waraos $(91,02 \pm 11,50 \mathrm{~cm})$ según Brito et al 2013, así como el obtenido por Bermúdez et al $2009^{15}$ en indígenas Añu $(89,74 \pm 15,43 \mathrm{~cm})$, lo cual es criterio diag- 
Figura 1

Frecuencia de componentes del síndrome metabólico según criterios de la IDF, ALAD y ATP III, de acuerdo a género. Indígenas de la etnia Kariña. Mayagua, Municipio Heres, Estado Bolívar - Venezuela, Mayo 2013 - Mayo 2014.

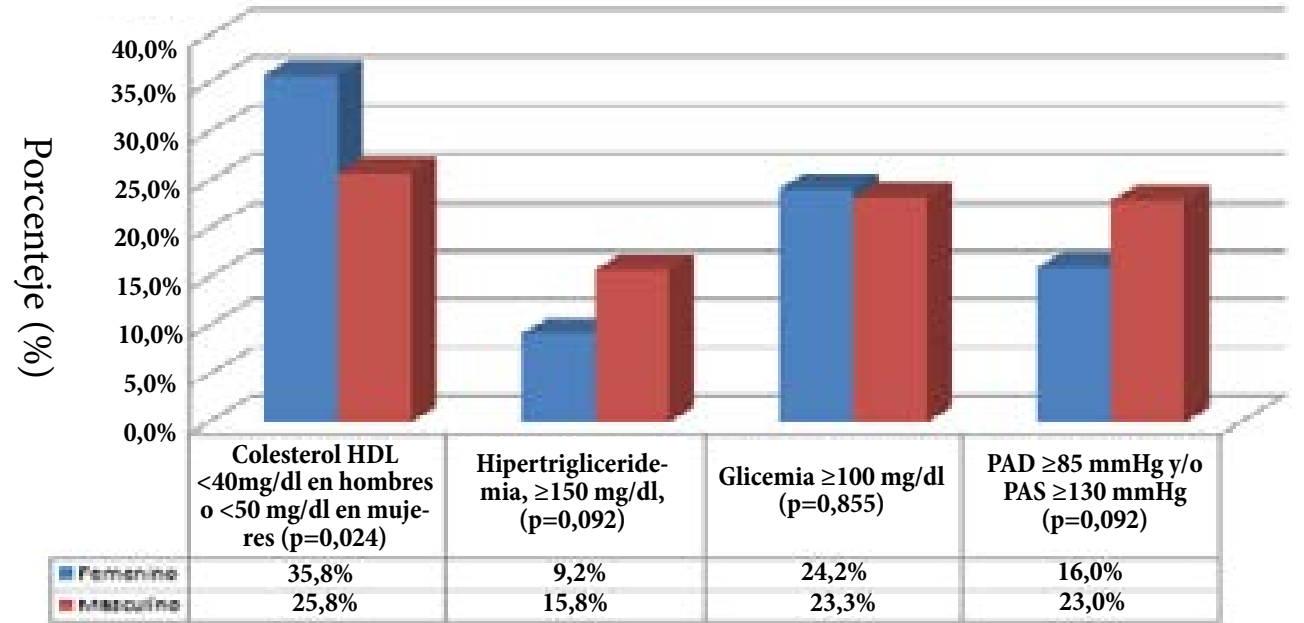

Fuente: Datos obtenidos de historia clínica realizada a la muestra de la investigación en la Comunidad de Mayagua.

Tabla 2 Frecuencia de síndrome metabólico según criterios de la IDF, ALAD y ATP III, de acuerdo a grupo etario. Indígenas de la etnia Kariña. Mayagua, Municipio Heres, Estado Bolívar - Venezuela, Mayo 2013 - Mayo 2014.

\begin{tabular}{|c|c|c|c|c|c|c|c|c|}
\hline \multirow{2}{*}{\multicolumn{2}{|c|}{ IDF }} & \multicolumn{2}{|c|}{$\begin{array}{l}\text { No Cumple Criterios para } \\
\text { Síndrome Metabólico }\end{array}$} & \multicolumn{2}{|c|}{ Síndrome Metabólico } & \multicolumn{2}{|l|}{ Total } & \multirow{2}{*}{$p$} \\
\hline & & $\mathrm{N}$ & $\%$ & $\mathrm{~N}$ & $\%$ & $\mathrm{~N}$ & $\%$ & \\
\hline \multirow{6}{*}{ Edad } & $18-29$ & 24 & 20,0 & 8 & 6,7 & 32 & 26,7 & \multirow{6}{*}{0,02} \\
\hline & $30-39$ & 18 & 15,0 & 13 & 10,8 & 31 & 25,8 & \\
\hline & $40-49$ & 8 & 6,7 & 10 & 8,3 & 18 & 15,0 & \\
\hline & $50-59$ & 7 & 5,8 & 12 & 10,0 & 19 & 15,8 & \\
\hline & 60 y más & 7 & 5,8 & 13 & 10,8 & 20 & 16,7 & \\
\hline & Total & 64 & 53,3 & 56 & 46,7 & 120 & 100,0 & \\
\hline \multirow{2}{*}{\multicolumn{2}{|c|}{ ALAD }} & \multicolumn{2}{|c|}{$\begin{array}{l}\text { No Cumple Criterios para } \\
\text { Síndrome Metabólico }\end{array}$} & \multicolumn{2}{|c|}{ Síndrome Metabólico } & \multicolumn{2}{|l|}{ Total } & \multirow{2}{*}{$\mathrm{p}$} \\
\hline & & $\mathrm{N}$ & $\%$ & $\mathrm{~N}$ & $\%$ & $\mathrm{~N}$ & $\%$ & \\
\hline \multirow{6}{*}{ Edad } & $18-29$ & 25 & 20,8 & 7 & 5,8 & 32 & 26,7 & \multirow{6}{*}{0,045} \\
\hline & $30-39$ & 21 & 17,5 & 10 & 8,3 & 31 & 25,8 & \\
\hline & $40-49$ & 10 & 8,3 & 8 & 6,7 & 18 & 15,0 & \\
\hline & $50-59$ & 8 & 6,7 & 11 & 9,2 & 19 & 15,8 & \\
\hline & 60 y más & 9 & 7,5 & 11 & 9,2 & 20 & 16,7 & \\
\hline & Total & 73 & 60,8 & 47 & 39,2 & 120 & 100,0 & \\
\hline \multirow[t]{2}{*}{ ATP III } & & \multicolumn{2}{|c|}{$\begin{array}{l}\text { No Cumple Criterios para } \\
\text { Síndrome Metabólico }\end{array}$} & \multicolumn{2}{|c|}{ Síndrome Metabólico } & \multicolumn{2}{|l|}{ Total } & \multirow{2}{*}{$\mathrm{p}$} \\
\hline & & $\mathrm{N}$ & $\%$ & $\mathrm{~N}$ & $\%$ & $\mathrm{~N}$ & $\%$ & \\
\hline \multirow{6}{*}{ Edad } & $18-29$ & 25 & 20,8 & 7 & 5,8 & 32 & 26,7 & \multirow{6}{*}{0,016} \\
\hline & $30-39$ & 23 & 19,2 & 8 & 6,7 & 31 & 25,8 & \\
\hline & $40-49$ & 9 & 7,5 & 9 & 7,5 & 18 & 15,0 & \\
\hline & $50-59$ & 8 & 6,7 & 11 & 9,2 & 19 & 15,8 & \\
\hline & 60 y más & 9 & 7,5 & 11 & 9,2 & 20 & 16,7 & \\
\hline & Total & 74 & 61,7 & 46 & 38,3 & 120 & 100,0 & \\
\hline
\end{tabular}

Fuente: Datos obtenidos de historia clínica realizada a la muestra de la investigación en la Comunidad de Mayagua. 


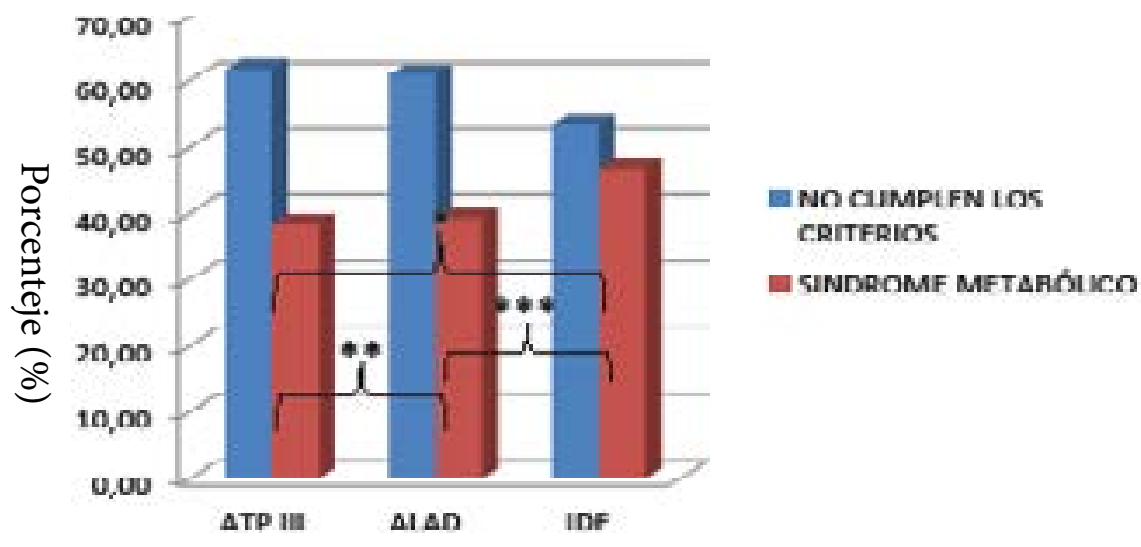

* Indice de Kappa (k) entre ATP III e IDF = 0,797

** Índice de Kappa (k) entre ATP III y $A L A D=0,912$

*** Índice de Kappa (k) entre ALAD e IDF = 0,848

Fuente: Datos obtenidos de historia clínica realizada a la muestra de la investigación en la Comunidad de Mayagua.

nóstico de obesidad abdominal según los criterios de la IDF, ATP III y ALAD ${ }^{11}$. Los aumentos relativos en el tejido adiposo visceral en comparación con el subcutáneo, con aumento de la circunferencia abdominal en asiáticos e hindúes asiáticos, pudiera explicar la prevalencia mayor del síndrome en tales poblaciones, en comparación con varones afroamericanos en quienes predomina la grasa subcutánea ${ }^{16}$.

De acuerdo a la concentración de HDL se obtuvo una media aritmética de 43,05 $\pm 9,66 \mathrm{mg} / \mathrm{dl}$, siendo mayor al obtenido por Brito, et al $2013^{8}(41 \pm 12,21 \mathrm{mg} / \mathrm{dl})$ y Bermúdez, et al $2009^{15}(39,1 \pm 10,6 \mathrm{mg} / \mathrm{dl})$, constituyendo HDL bajo como criterio diagnóstico para síndrome metabólico según los criterios utilizados. En lo que respecta a los triglicéridos se encontró una media aritmética de 130,98 $\pm 116,55 \mathrm{mg} / \mathrm{dl}$, menor a la descrita por Brito, et al $2013^{8}(135,05 \pm 65,70 \mathrm{mg} /$ dl). La media de glicemia en ayuna es $102,45 \pm 19,03 \mathrm{mg} / \mathrm{dl}$ constituyendo criterio diagnóstico para síndrome metabólico ${ }^{11}$, siendo mayor a la obtenida por Brito, et al $2013^{8}$ $(82,89 \pm 26,24 \mathrm{mg} / \mathrm{dL})$.

Se evidencia un promedio para la PAS 120,75 $\pm 14,68$ $\mathrm{mmHg}$ y $\mathrm{PAD} 81,17 \pm 13,36 \mathrm{mmHg}$, similar a la reportada por Brito, et al 2013 (PAS 123,28 $\pm 15,27$ mmHg; PAD $78,11 \pm 9,67 \mathrm{mmHg}^{8}$.

La obesidad abdominal es frecuente en el género femenino según ALAD (género femenino 31\% y masculino 28\%) y ATP III (género femenino 31\% y masculino 16\%) semejante a lo reportado por Brito, et al ${ }^{8}$ (ALAD: género femenino 55,9\% y masculino 51,9\%; ATP III: género femenino 61,8\% y masculino 22,2\%), en cambio según la IDF el género masculino fue más afectado (género masculino 39\% y femenino 38\%), lo cual difiere al estudio de Brito, et $\mathrm{al}^{8}$ (género femenino 91,2\% y masculino 55,6\%). El Estudio Strong Heart demostró que la comunidad indígena Pima presenta mayor riesgo de obesidad ya que más del $50 \%$ de la población adulta es obe$\mathrm{sa}^{17}$. En estudios previos en Chile se describió un incremento gradual en la prevalencia de obesidad en individuos de la etnia Mapuche en el área urbana, diferenciándose de las rurales, lo que puede deberse al proceso de adaptación de los comportamientos propios de su forma de vida a los hábitos modernos $^{13}$. En el estudio científico Evaluación Múltiple de Riesgo Cardiovascular en América Latina (CARMELA) en el 2008 se reportó que la prevalencia de obesidad abdominal aumentó con la edad, hallazgo similar al de este estudio ${ }^{18}$.

La obesidad podría ser una de las causas fundamentales de la alta prevalencia de síndrome metabólico ya que se incrementa simultáneamente con el aumento de la obesidad ${ }^{14}$. El tejido adiposo visceral o abdominal es activo en liberación de ácidos grasos, factor de necrosis tumoral a (TNF-a), leptina, resistina, Interleucina-6 (IL-6) y otras sustancias capaces de promover el desarrollo de estado proinflamatorio, daño endotelial y/o resistencia a la insulina, porque produce un cambio en la sensibilidad de la insulina en el tejido muscular debido al incremento de ácidos grasos circulantes que generan inhibición de la captación de glucosa, además da lugar al aumento en la formación de triglicéridos, aumento de gluconeogénesis, aumento de VLDL, aumento de LDL y disminución de $\mathrm{HDL}^{19,20}$. La liberación de ácidos grasos se debe a que las catecolaminas mediante el receptor $\beta 3$ tienen acción lipolítica a nivel del tejido adiposo visceral ${ }^{21}$. Por otro lado, la liberación del TNF-a permite mantener la concentración de ácidos grasos circulantes elevados, debido a que este promueve la lipólisis e inhibe la lipogénesis, dando lugar a la alteración de la sensibilidad a la insulina ${ }^{22}$.

En la hiperglicemia se encontró una frecuencia de 47,50\% siendo más elevada a la comunicada por Bermúdez, et al $2009^{15}$ (14\%) y Brito, et al $^{8} 2013$ (9,8\%). Algunos estudios en poblaciones indígenas de México informan la prevalencia de diabetes mellitus 4,4\% en indígenas otomíes de Querétaro; en tribus Pima de Sonora de hombres 6,3\% y mujeres 10,5\%; en mazatecas de Oaxaca 2,1\%, y otros tipos de trastornos metabólicos en poblaciones tepehuana, huichol y mexicanera de Durango; mayas de Yucatán y triquis de Oaxaca ${ }^{23,24}$. 
La prevalencia de síndrome metabólico en indígenas Kariña es mayor a la reportada en Waraos $^{8}$ y Añú ${ }^{15}$, es frecuente en el género femenino, hallazgo constante en $A \tilde{n} u^{15}$. Waraos $^{8}$, estudio CARMELA ${ }^{18}$ y en una revisión sistemática realizada por De Carvalho, et al en adultos brasileños en el que se determinó mayor prevalencia síndrome metabólico $(65,3 \%)$ en un estudio realizado en la población indígena de Río Grande do Sul, utilizando como criterio diagnóstico el NCEP-ATP III (2001) ${ }^{25}$. Gyakobo, et al realizó un estudio en una población rural de Ghana en el cual la prevalencia síndrome metabólico según los criterios de la IDF y NCEP ATP III fue frecuente en mujeres lo que coincide con esta investigación ${ }^{26}$. Según Misra, et al la prevalencia de síndrome metabólico en Sri Lanka es alta en la cual el 35\% corresponde a hombres y el $51 \%$ en mujeres, lo que pone de manifiesto una vez más la frecuencia en el género femenino ${ }^{27}$. En China prevalencia es muy baja entre las mujeres jóvenes y aumenta en mujeres de mediana edad y de edad avanzada ${ }^{14}$. El síndrome metabólico es prevalente entre los andinos del Perú, especialmente en mujeres mayores descrito por Medina-Lezama, et $\mathrm{al}^{28}$.

El síndrome metabólico fue frecuente en edades mayores similar a lo reportado por CARMELA ${ }^{18}$, Bermúdez, et $\mathrm{al}^{15}{ }^{15}$ Brito, et $\mathrm{al}^{8}$ y De Carvalho, et $\mathrm{al}^{25}$. Se ha demostrado que en varias partes del mundo la prevalencia de dicha entidad clínica está aumentando, y se estima en alrededor de $20-25 \%$ de la población adulta, resultado de un incremento de la obesidad y vida sedentaria ${ }^{25}$. La prevalencia general del síndrome metabólico es de $24 \%$ en poblaciones de origen caucásico, la cual aumenta en función de la edad a más del $30 \%$ en personas mayores de 50 años y más del $40 \%$ en mayores de 60 años ${ }^{15}$.

La presencia de resistencia a la insulina en pacientes con síndrome metabólico aumenta el riesgo cardiovascular, debido a que las VLDL se hacen más aterogénicas por el aumento de ésteres de colesterol y las LDL también adquieren esta propiedad por su modificación oxidativa, capacidad de acumularse en la íntima arterial y ser captadas por los receptores de macrófagos, lo cual facilita la formación de aterosclerosis ${ }^{29,30}$.

En conclusión, se encontró una alta prevalencia de Síndrome Metabólico tanto por los criterios diagnósticos de ALAD, IDF y ATP III, el cual predomina en el género femenino y en individuos mayores de 50 años, por lo que presentan mayor riesgo de presentar enfermedades cardiovasculares y diabetes, motivo por el cual se recomienda la realización de este tipo de investigación en etnias indígenas, con la finalidad de documentar los factores de riesgos, cambios en el estilo de vida y hábitos alimenticios que desencadenen el desarrollo de Síndrome Metabólico, enfermedades cardiovasculares y Diabetes Mellitus; de esta forma promover y fomentar la promoción de la salud, así como también el tratamiento oportuno para mejorar la calidad de vida de dichas poblaciones indígenas.

\section{REFERENCIAS}

1.Rojas E, Velasco M, Bermúdez V, Israili Z, Bolli P. Targeting Hypertension in Patients with Cardiorenal Metabolic Syndrome. Curr Hypertens Rep [Internet].2012 [Citado Marzo 2014];11(1):1-9. Disponible en: https://www.researchgate.net/ publication/230588683_Targeting_Hypertension_in_Patients_with_ Cardiorenal Metabolic Syndrome

2.Bermúdez V, París Marcano R, Cano C, Arráiz N, Amell A, Cabrera M, et al. The Maracaibo City Metabolic Syndrome Prevalence Study: Design and Scope. American Journal of Therapeutics [Internet].2010 [Citado en Marzo 2014];17:288-294. Disponible en: https://www.researchgate.net/publication/41001103 The Maracaibo_City_Metabolic_Syndrome_Prevalence_Study_Design_and Scope

3.López ME, Sosa MA, Labrousse NPM. Síndrome Metabólico. Rev. posgrado VIa. Cátedra Med [Internet].2007 [Citado en Enero 2014];174:12-15. Disponible en: http://med.unne.edu.ar/revistal revista174/3 174.pdf

4.Crepaldi $\overline{\mathrm{G}}$, Maggi S. El Síndrome Metabólico: contexto histórico. Diabetes Voice [Internet]. 2006 [Citado en Enero 2014]; 51:8-10. Disponible en: https://scholar.google

$\mathrm{cl} /$ scholar? cluster $=16325125827136121768 \& h l=e s \& a s \_s d t=0,5$ 5.Jurado Santa Cruz F, Peralta Cordero G, Morales Sánchez M, Rodríguez Acar M, Peralta Pedrero ML. Psoriasis y síndrome metabólico. Rev Cent Dermatol Pascua [Internet].2013;22(2):50Disponible en: http://www.medigraphic.com/pdfs/derma/cd-2013/ cdl32b.pdf

6.Lizarzaburu Robles JC. Síndrome metabólico: concepto y aplicación práctica. An Fac med [Internet].2013 [Citado en Abril 2014];74(4):315-320. Disponible en: http://www.scielo.org.pe/pdf/ afm/v74n4/a09v74n4.pdf

7.Ríos García AL, Alonso L M, Carmona Z, Cabana Jiménez AD, Martínez Orellano R. Frecuencia y factores de riesgo para el desarrollo del síndrome metabólico en pacientes del programa de obesidad de una institución de salud en Barranquilla (Colombia), 2011. Salud Uninorte Barranquilla [Internet].2013 [Citado en Enero 2014];29(2):315-326. Disponible en: http://www.redalyc.org/ pdf/817/81730430016.pdf

8.Brito N, Córcega A, Marín M, Bognanno JF, Alcázar RJ, Pérez $\mathrm{K}$. Frecuencia de Síndrome Metabólico en Indígenas de la Etnia Warao de Barrancas del Orinoco, Estado Monagas. Venezuela. Rev Venez Endocrinol Metab [Internet].2013 [Citado en Enero 2014];11(3):128-140. Disponible en: http://www.saber.ula.ve/ bitstream/123456789/37945/1/articulo3.pdf

9.Viso M, Rodríguez Z, Aponte L, Barboza A, Barreto P, Villamizar $\mathrm{M}$, et al. Insulinorresistencia, obesidad y síndrome metabólico. Cohorte CDC de Canarias en Venezuela. Salus [Internet].2013 [Citado en Enero 2014];17(1):18-24. Disponible en: http://www. redalyc.org/ $\mathrm{html} / 3759 / 375933972005 /$

10.Freire G, Villalón ME, Biord H, Scaramelli F, Tarble K, Perera MA, et al. Salud indígena en Venezuela. Ministerio del Poder Popular para la Salud [Internet]. 2007 [Citado en 2014];(2):7239. Disponible en: https://books.google.cl/books?hl=es\&lr=\&id= $x 1$ LIKgjetk YC\&oi $=$ fnd $\& p g=\quad P R 2 \& d q=$ salud + indigena + en + venezuela\&ots $=Z$ s g nqe 1 wMA\&sig $=$ gj C $9 S y Q$ $V s R B A X j V C N H 8$ tbmxgv0\#v=onepage $\& q=$

salud\%20indigena\%20en\%20venezuela $\& f=$ false

11. Sinay I, Costa Gil J, De Loredo L, Ramos O, Lúquez H, Da Silva Filho RL, et al. Epidemiologia, Diagnostico, Control, Prevención y Tratamiento del Síndrome Metabólico en Adultos. Consenso Latinoamericano de la Asociación Latinoamericana de Diabetes (ALAD) [Internet].2010 [Citado en Marzo 2014];XVIII(1):25-44. Disponible en: http://www.revistaalad.com/

pdfs/100125-44.pdf

12.Landis JR, Koch GG. The Measurement of Observer Agree- 
ment for Categorical Data. International Biometric Society[Internet].2013 [Citado en Noviembre 2014];33(1):159-174. Disponible en: http://www.jstor.org/stable/2529310

13.Philco P, Serón P, Muñoz S, Navia P, Lanas F. Factores asociados a síndrome metabólico en la comuna de Temuco, Chile. Rev Med Chile [Internet].2012 [Citado en Marzo 2014];140:334-339. Disponible en: http://www.scielo.cl/pdf/rmc/v140n3/art08.pdf

14.Cai H, Huang J, Xu G, Yang Z, Liu M, et al. Prevalence and Determinants of Metabolic Syndrome among Women in Chinese Rural Areas. PLoS ONE [Internet].2012 [Citado en Noviembre 2014];7(5):1-1. Disponible en: http://journals.plos.org/plosone/ article? id=10.1371/journal.pone.0036936

15.Bermúdez P, Valmore J, Finol G, Freddy J, Leal N, Parra V, et al. Prevalencia del síndrome metabólico en la población adulta Añú de la laguna de Sinamaica del municipio Páez, estado Zulia. Revista Latinoamericana de Hipertensión. [Internet].2009 [Citado en Marzo 2014];4(3):63-70. Disponible en: http://www.redalyc.org/ pdf/1702/170216837002.pdf

16.Eckel RH. Síndrome metabólico. En: Fausi AS, Braunwald E, Kasper DL, Hauser EL, Longo DL, Jameson JL, et al. Harrison Principios de Medicina Interna. 17a ed. México: McGraw-Hill Interamericana; 2009.p.1509-1514

17.Chacín M, Rojas J, Pineda C, Rodríguez D, Núñez Pacheco M, Márquez Gómez M, et al. Predisposición humana a la Obesidad, Síndrome Metabólico y Diabetes: El genotipo Ahorrador y la incorporación de los diabetogenes al genoma humano desde la Antropología Biológica. Síndrome Cardiometabólico [Internet].2011 [Citado en Marzo 2014];1(1):11-24. Disponible en: https:// $w w w$.researchgate.net/publication/249011664 Predisposicion humana a la Obesidad Sindrome Metabolico y Diabetes_El genotipo Ahorrador y la incorporacion de los diabetogenes al genoma humano desde la Antropologia Biologica

18. Escō̄edo J, Schargrodsky $\mathrm{H}$, Champagne B, Silva H, Boissonnet CP, Vinueza R, et al. Prevalence of the Metabolic Syndrome in Latin America and its association with subclinical carotid atherosclerosis: the CARMELA cross sectional study. Cardiovascular Diabetology [Internet].2009 [Citado en Marzo 2014];8:52. Disponible en: https://www.researchgate.net/ publication/26837956 Prevalence of the Metabolic Syndrome in Latin America and its association with sub-clinical carotid atherosclerosis The CARMELA cross sectional study

19.Albornoz López R, Pérez Rodrigo I. Nutrición y síndrome metabólico. Nutr clín diet Hosp [Internet].2012 [Citado en Enero 2014];32(3):92-97. Disponible en: http://www.nutricion.org/ publicaciones/revista 201232 3/NUTRICION.pdf

20.BrescianiSalaroli $\overline{\mathrm{L}}$, Dias Săliba RA, Zandonade E, Bisi Molina $M$, Souza Bissoli N. Prevalence of metabolic syndrome and related factors in bank employees according to different defining criteria, Vitória/ES, Brazil. CLINICS [Internet].2013 [Citado en Noviembre 2014];68(1):69-74. Disponible en: https://www.ncbi.nlm. nih.gov/pmc/articles/PMC3552453/
21.Rojas J, Bermudez V, Leal E, Aparicio D, Peña G, Acosta 1, et al. Origen étnico y enfermedad cardiovascular. Archivos Venezolanos de Farmacología y Terapéutica [Internet].2008 [Citado en Marzo 2014];27(1):41-58. Disponible en: https://www.researchgate. net/publication $/ 233967188$ Origen etnico y enfermedad cardiovascular

22.Acosta García E. Vigencia del Síndrome Metabólico. Acta bioquím clín Latinoam [Internet].2011 [Citado en Enero 2014];45(3):423-430. Disponible en: http://www.redalyc.org/ $p d f / 535 / 53521520003 . p d f$

23.Bojorges Velázquez LA, Castillo Herrera CJA, Jiménez Tamayo R. Factores de riesgo de síndrome metabólico en estudiantes de la universidad Pablo Guardado Chávez, año 2013. Rev Cubana Invest Bioméd [Internet].2013 [Citado en Marzo 2014];32(4):379388. Disponible en: http://scielo.sld.cu/scielo.php?pid=S0864$03002013000400001 \&$ script $=s c i$ arttext $\&$ tlng $=p t$

24.Herrera Huerta EV, García Montalvo EA, Méndez Bolaina E, López López JG, Valenzuela OL. Sobrepeso y obesidad en indígenas nahuas de Ixtaczoquitlán, Veracruz, México. Rev Perú Med Exp Salud Pública [Internet].2012 [Citado en Marzo 2014];29(3):345-349. Disponible en: http://www.scielo.org.pe/pdf/rins/v29n3/a08v29n3.pdf 25.De Carvalho Vidigal F, Bressan J, Babio N, Salas Salvadó $\mathrm{J}$. Prevalence of metabolic syndrome in Brazilian adults: a systematic review. BMC Public Health [Internet].2013 [Citado en Noviembre 2014];13:1198. Disponible en: http://www.biomedcentral. com/1471-2458/13/1198

26.Gyakobo M, Amoah A, Martey Marbell D, Snow R. Prevalence of the metabolic syndrome in a rural population in Ghana. BMC Endocrine Disorders [Internet].2012 [Citado en Noviembre 2014];12:25. Disponible en: http://www.biomedcentral.com/1472$6823 / 12 / 25$

27.Misra A, Misra R, Wijesuriya M, Banerjee D. The metabolic syndrome in South Asians: Continuing escalation \& possible solutions. Indian J Med Res [Internet].2007 [Citado en Noviembre 2014];125:345-354. Disponible en: http://icmr.nic.in/ijmr/2007/ March/0310.pdf

28.Medina Lezama J, Zea Diaz H, Morey Vargas O, Bolaños Salazar JF, Muñoz Atahualpa E, et al. Prevalence of the metabolic syndrome in Peruvian Andean hispanics: The PREVENCION study. Elsevier [Internet].2007 [Citado en Noviembre 2014];78:270 281. Disponible en: https://scholar.google.cl/scholar?cluster= $14081083358573048114 \& h l=e s \&$ as $s d t=0,5$

29.Soca PEM. Mecanismos del riesgo cardiovascular en el síndrome metabólico. Rev Fed Arg Cardiol [Internet].2013 [Citado en Febrero 2014];42(3):166-167. Disponible en: http://www.fac.org. ar/1/revista/13v42n3/editor/edit02/soca.pdf

30.Fernández SL, Rueda Clausen CF, Pradilla LP, López Jaramillo P, Lahera V. Participación de la Angiotensina II en el desarrollo de la enfermedad aterosclerótica. Rev. Med [Internet].2006 [Citado en Marzo 2014];14(1):8-18. Disponible en: http://www.redalyc.org/ $\mathrm{html} / 910 / 91014103 /$ 